\title{
Optimization of vehicle compartment low frequency noise based on Radial Basis Function Neuro-Network Approximation Model
}

\author{
Qi-guo $H U^{l}, Y u$-qian $W A N G^{1} *$ Su-ping $L I^{2}$ \\ ${ }^{1}$ Department of Mechanical \& Vehicle Engineering, Chongqing Jiaotong University, Chongqing, 400074, China \\ ${ }^{2}$ Department of Traffic \& Transportation, Chongqing Jiaotong University, Chongqing, 400074, China
}

\begin{abstract}
For reducing the vehicle compartment low frequency noise, the Optimal Latin hypercube sampling method was applied to perform experimental design for sampling in the factorial design space. The thickness parameters of the panels with larger acoustic contribution was considered as factors, as well as the vehicle mass, seventh rank modal frequency of body, peak sound pressure of test point and sound pressure root-mean-square value as responses. By using the RBF(radial basis function) neuro-network method, an approximation model of four responses about six factors was established. Further more, error analysis of established approximation model was performed in this paper. To optimize the panel's thickness parameter, the adaptive simulated annealing algorithm was im-plemented. Optimization results show that the peak sound pressure of driver's head was reduced by $4.45 \mathrm{~dB}$ and $5.47 \mathrm{~dB}$ at frequency $158 \mathrm{HZ}$ and $134 \mathrm{~Hz}$ respec-tively. The test point pressure were significantly reduced at other frequency as well. The results indicate that through the optimization the vehicle interior cavity noise was reduced effectively, and the acoustical comfort of the vehicle was im-proved significantly.
\end{abstract}

\section{INTRODUCTION}

In addition to focus on the stability and safety of driving, customers pay more attention to the acoustic characteristics of vehicle compartment. One of the main reasons that affect the acoustic performance of the cab is the prominent low frequency noise which is difficult to be eliminated [1-3]. Previous investigation shows that the thin plates' structure radiation noise which is generated by external excitation in the frequency range of $0 \mathrm{~Hz} \sim 200 \mathrm{~Hz}$ contributes significantly to the low frequency noise of the $\mathrm{cab}[1]$. In order to reduce the noise in cab and improve vehicle acoustical comfort, the body structure that contributes tremendously to the acoustic should be optimized. The finite element calculation method for optimization is time consuming in view of the model size. Comparatively, the approximation model optimization method is of high efficiency[1-3]. In paper [3], the thickness of plate was designated as the design variable. The minimum pressure at the driver's right ear and minimum body mass was defined as the optimization objective. The approximation model was established by response surface method to optimize the acoustic performance. In paper [4], the second response surface approximation model was established for the optimization of acoustic and vibration. The method of using response surface method to establish the approximation model has the characteristics of simplicity expression and high computational efficiency. However, it fails to guarantee the accuracy of the model while the number of samples is limited.

This paper is organized as follows. In section 2, considering the low frequency noise of the cab, the optimal Latin Hypercube Sampling (LHS) method was used to collect sample data and the RBF neural network method was implemented to establish the approximation model. In this way the approximation model of high accuracy can be established with a small amount of sample data and the acoustic optimization efficiency can be improved. In section 3, based on the established approximation model, the adaptive simulated annealing algorithm was used to optimize the thickness parameters of the car body to improve the acoustic performance. In section 4 , the simulation experiment results of vehicle compartment low frequency noise is conducted and more details are analyzed. In section 5, the conclusion is illustrated.

\section{MODELING}

\subsection{Finite Element Model}

The complex car body structure is composed of thin plates. The finite element model was simplified and meshed by shell element with the premise of assuring the main body structure's characteristics. Considering the calculation precision and the computation burden, the size of the grid unit is designated as $10 \mathrm{~mm}$. The closed chamber of the car body was meshed by the tetrahedral element. As the seats influenced on the acoustic characteristics of cavity sound field[3], the seat model was also established while establishing the finite element model. Based on the principle that the acoustic grid unit length contain at least six units in each wavelength, the grid unit length is designated as $50 \mathrm{~mm} \sim 140 \mathrm{~mm}$. The established finite element model of cavity sound field is shown in Figure 1, which contains 29467 tetrahedral elements and 6229 nodes, as the car body structure is composed of 444150 units and 464592 nodes.

*Corresponding author:haqiankaka@163.com 


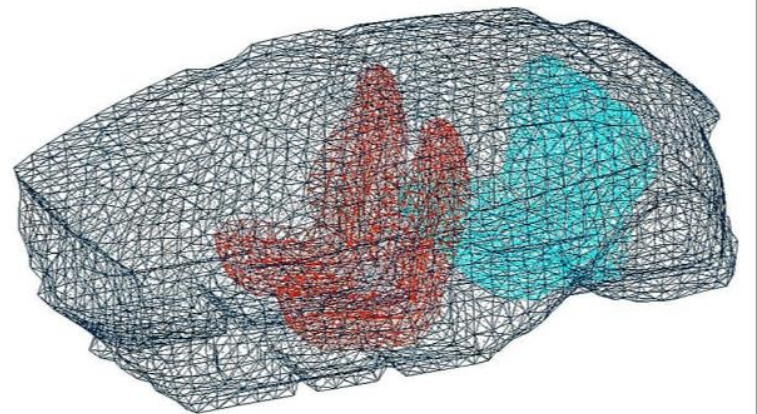

Fig. 1. Finite element model of cavity sound field

\subsection{Approximation Model}

The sound pressure response output point was defined at the driver's head $\mathrm{D}$, deputy driver's head $\mathrm{P}$, left and right rear passenger head RL, RR[6]. The engine force at $0 \mathrm{~Hz}-300 \mathrm{~Hz}$ and the suspension force caused by the random road incentive force was loaded at the same time as the external excitation. Through the finite element simulation, the A-level weighting acoustic pressure curve at test point is shown in Figure 2 .

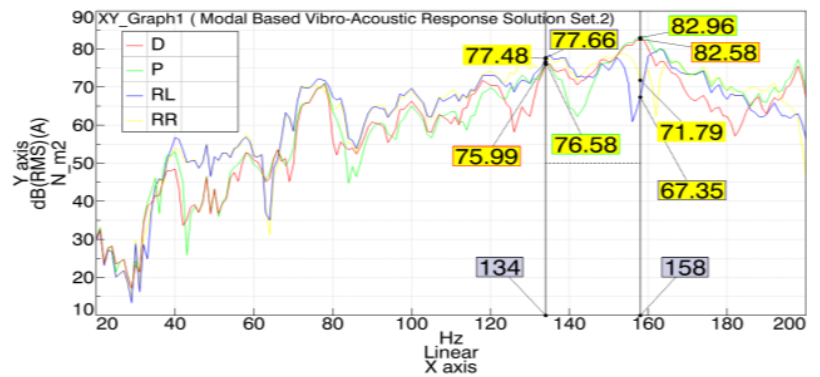

Fig. 2. A-level weighting acoustic pressure curves of four test point location

Based on figure 2, the response analysis of acoustic coupling based on the mode shows that at the low frequency of $0 \mathrm{~Hz}-200 \mathrm{~Hz}$, the sound pressure of cab test points is high near $158 \mathrm{~Hz}, 134 \mathrm{~Hz}, 198 \mathrm{~Hz}, 152 \mathrm{~Hz}$ and $78 \mathrm{~Hz}$.

\subsubsection{Collection of sample data}

The analysis of the panel acoustic contribution[9] shows that where sound pressure frequency is high, the body floor, the ceiling around, the front door and the inner panel contribute mainly to acoustics performance of test point. In order to reduce the pressure in cab and avoid the excessive increase of body mass as well as the seventh rank modal frequency, the body mass, the seventh rank modal frequency, the sound pressure peak value and the RMS(root mean square) of sound pressure at the driver's head were taken as the response of test design. Each group's level value at test design matrix is designated as input parameter.

In the sampling experiment, the thickness parameter of cab floor $\left(t_{1}\right)$,rear floor $\left(t_{2}\right)$, the front panel $\left(t_{3}\right)$, ceiling $\left(t_{4}\right)$, front door inner plate $\left(t_{5}\right)$ and rear door inner plate $\left(t_{6}\right)$ were considered as a factor, whose design range is $0.5 \mathrm{~mm} \leq \mathrm{ti} \leq 2 \mathrm{~mm}$. In order to establish the accurate approximation model by less sampling point, the optimal Latin Hypercube Sampling(LHS) method was used for test designing[10]. Taking 30 levels for each factor in the range, there are 30 level assemblies of design matrix through opt-LHS.

The optimal LHS improved the weakness of random LHS. It has the ability to allocate all test points welldistributed in design space. It has excellent filling property and proportionality. It can achieve a high precise between response and factor.

\subsubsection{RBF(radial basis function) neural network approximation model}

Based on the data achieved in part 1, six factors were designated as the input parameter and four responses were designated as output parameter. The approximation model was established by RBF neural network. Compared with other approximation model, the RBF neural network model has strong nonlinear approximation ability. It doesn't rely on mathematical assumptions. More over, it has the most optimal approximation ability for functions and can approximate any continuous function at any accuracy.

Taking the peak sound pressure $D$ of the approximation model plate thickness $t_{1}, t_{2}, t_{3}, t_{4}, t_{5}, t_{6}$ as the example, the part of the three-dimensional surface is shown in figure 3.

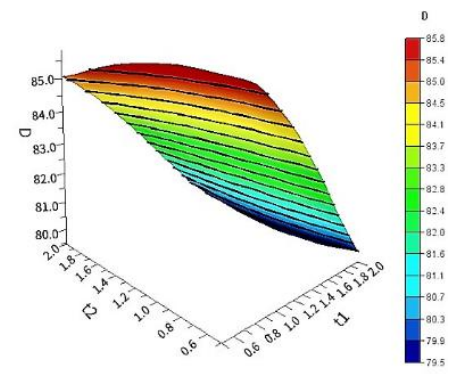

(a) $D-t_{1}-t_{2}$

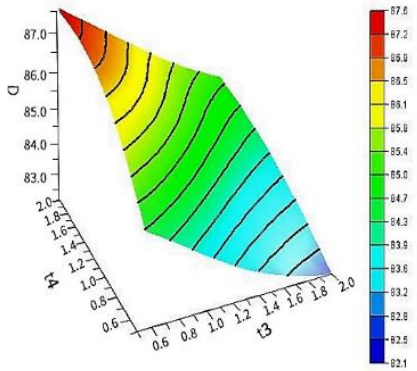

(b) $D-t_{3}-t_{4}$

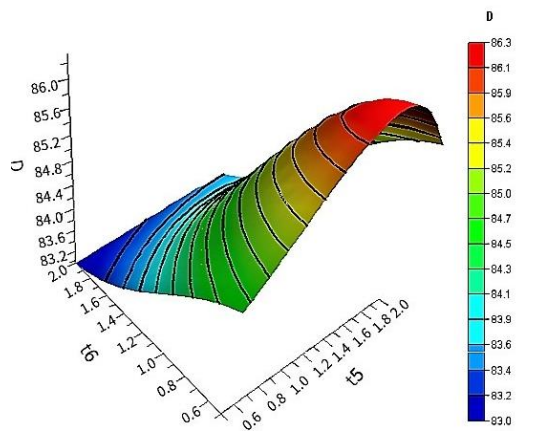

(c) $D-t_{5}-t_{6}$

Fig. 3. Approximation model surface of pressure peak

\subsubsection{Error Analysis}

In order to estimate the accuracy and reliability of the RBF neural network approximation model, 20 noninterpolation points in the design variable space were used as error checking samples to analyze the error of the approximation model. The accuracy of the model is evaluated by four indexes: mean error, maximum error, RMS error and multiple correlation coefficients. The requested value range is listed in table 1 .

Table 1.The requested value range 


\begin{tabular}{|c|c|c|}
\hline Mean error & 0 & 0.2 \\
\hline Max error & 0 & 0.3 \\
\hline RMS error & 0 & 0.2 \\
\hline $\begin{array}{c}\text { Mutli-correlation } \\
\text { coefficient }\end{array}$ & 0.9 & 1 \\
\hline
\end{tabular}

The error analysis results of the RBF neural network model are shown in Table 2.

Table 2. Error analysis index

\begin{tabular}{|c|c|c|c|c|}
\hline & mean error & $\begin{array}{c}\text { maximum } \\
\text { error }\end{array}$ & $\begin{array}{c}\text { RMS } \\
\text { error }\end{array}$ & $\begin{array}{c}\text { multiple } \\
\text { correlation } \\
\text { coefficient }\end{array}$ \\
\hline$D$ & 0.01153 & 0.03033 & 0.01423 & 0.9976 \\
\hline$d r m s$ & 0.01383 & 0.04563 & 0.01816 & 0.099608 \\
\hline$m$ & 0.01413 & 0.03206 & 0.01729 & 0.99531 \\
\hline$s f$ & 0.02195 & 0.06341 & 0.0259 & 0.9936 \\
\hline
\end{tabular}

According to the error analysis index, mean error, maximum error and the RMS error of approximation model are far less than the standard error. The multiple correlation coefficient of the output response is very close to 1 , which indicates that the model error is neglectable.

The corresponding input was designated as the input parameter of error check samples. All of the predicted value, such as the body mass, seventh rank modal frequency, peak pressure and acoustic pressure RMS at driver's head were calculated by approximation modal. The relationship between the true and predicted value of the four response points are shown in figure 4.

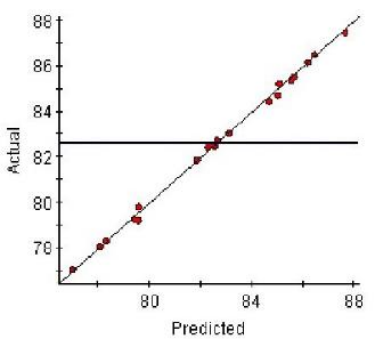

a) Sound pressure peak value

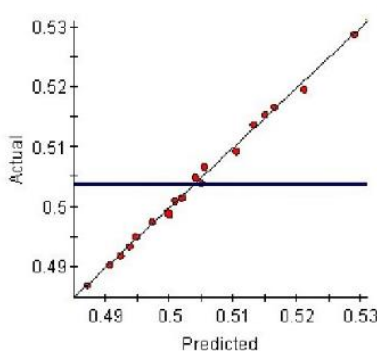

(c) body mass

Fig. 4. Relationship between the real value and the predicted value

As shown in Figure 4, there is tiny difference between the predicted value and the value calculated by the approximation model, which further proves that the model is of high accuracy.

Briefly, the sampling points were collected by optLHS test design. The approximation model was established by RBF neural network. The input parameter was designated as panel thickness. The output response was designated as the body weight, seventh rank modal frequency, the peak pressure and the acoustic pressure RMS at driver's head. Through the error analysis, the established approximation model has the characteristic of small error and high precision, which is able to meet the modeling requirements and replace the finite element model for optimization design.

\section{OPTIMIZATION}

\subsection{Mathematical Optimization Model}

Based on the established RBF neural network approximation model, the optimization objective function was created to minimum the peak value of sound pressure at driver's head. The design variables were defined as plate thickness parameters $t_{i}(1 \leqslant i \leqslant 6)$.

The RMS of sound pressure can reflect the overall level of cab pressure. In order to prevent the increase of cab sound pressure caused by the change of body structure, the RMS value of sound pressure at driver's head which is less than or equal to $60 \mathrm{~dB}$ was defined as one of the constraints.

The change of the plate thickness would lead to the change of the weight of the car body. Considering the requirement of light-weighted design, the weight should be limited within a certain range. In the original design, the body structure mass is 0.4802 t. In the optimization design, the body mass should be limited less than or equal to $0.5 \mathrm{t}$.

As the change of body plate thickness, seventh rank modal usually locates at the front door while sampling the seventh rank body modal frequency response, whose main frequency is $24 \mathrm{~Hz}$. However, there are several individual factor level combination conditions at which seventh rank modal frequency is too low. If the modal frequency of car door is too low, the car door will be easily affected by external load excitation which causes a high cab sound pressure. So the limitation of seventh rank modal frequency is:

$$
s f \cong 24 \mathrm{~Hz}
$$

$s f$ denotes car body seventh rank modal frequency.

The mathematical function of the optimization problem can be expressed as:

$$
\begin{aligned}
\min & D=f\left(t_{1}, t_{2}, t_{3}, t_{4}, t_{5}, t_{6}\right) \\
\text { s.t. } & \left\{\begin{array}{l}
d r m s \leq 60 \mathrm{~dB} \\
m \leq 0.5 \mathrm{t} \\
s f \geq 24 \mathrm{~Hz} \\
0.5 \mathrm{~mm} \leq t_{i} \leq 2 \mathrm{~mm}
\end{array}\right.
\end{aligned}
$$

\subsection{Optimization Theory}

Based on the plate thickness, adaptive simulated annealing algorithm was used for optimization. Adaptive simulated annealing (ASA) is an efficient global optimization algorithm for solving nonlinear optimization problems with multiple peaks and non- 
smoothness [12].

The way of implementing ASA algorithm is:

(I).Generate an initial solution $\omega \in W$ randomly. The Initial temperature $T_{\text {accept }}(0)$ of acceptance probability function equals $J(\omega)$. The initial temperature of generated probability function is defined as $T_{i, \text { gen }}(0)$, $1 \leq i \leq \mathrm{n}$. Both of them were set to 1 . The control parameter $c$ is given by the user. Annealing times $k_{i}(1 \leq i \leq \mathrm{n})$ and $k_{\alpha}$ are both set to 0 .

(II). The new solution is generated on solution space by following equation.

$$
\omega_{i}^{\text {new }}=\omega_{i}^{\text {old }}+q_{i}\left(U_{i}-L_{i}\right)
$$

Where, $1 \leq i \leq \mathrm{n}, \quad \omega^{\text {new }} \notin W$.

$$
q_{i}=\operatorname{sgn}\left(v_{i}-\frac{1}{2}\right) T_{i, \text { gen }}\left(k_{i}\right)\left[\left[1+\frac{1}{T_{i, g e n}\left(k_{i}\right)}\right]^{2 v_{i}-1 \mid}-1\right] \text {; }
$$

$v_{i}$ denotes the uniformly random distributed variables from $[0,1]$. If the new generated solution $\omega^{\text {new }} \notin W$, abandon and regenerate a new solution till it satisfies $\omega^{\text {new }} \in W$.

The acceptance probability function of new solution $\omega^{\text {new }}$ is determined by the following equation.

$$
\begin{aligned}
& P_{\text {accept }}=\frac{1}{1+\exp \left(\left(J\left(\omega^{\text {new }}\right)-J\left(\omega^{\text {old }}\right) / \mathrm{T}_{\text {accept }}\left(k_{\alpha}\right)\right)\right.} \\
& \approx \exp \left(-\Delta J / \mathrm{T}_{\text {accept }}\left(k_{\alpha}\right)\right)
\end{aligned}
$$

From the above we obtain that $\Delta \mathrm{J}=\mathrm{J}\left(\omega^{\text {new }}\right)-J\left(\omega^{\text {old }}\right)$. If $P_{\text {accept }} \geq P_{\text {unif }}$, the new solution is acceptable, otherwise abnegated. $P_{\text {unif }}$ represents the uniformly distributed variable in $[0,1]$.

(III).Every $N_{\text {accept }}($ th) times while the solution is accepted, tempering is processed. The following equation is implemented for calculating sensitivity of each variable, which describes the gradient variation.

$$
s_{i}=\frac{J\left(\omega^{\text {best }}+e_{i} \delta\right)-J\left(\omega^{\text {best }}\right)}{\delta}, 1 \leq i \leq \mathrm{n}
$$

Where $\omega^{\text {new }}$ is the optimal solution by now. $\delta$ is the step size. The $i$ (th) element in n-dimension vector $e_{i}$ is set to 1 , else 0 . Find $s_{\max }=\max \left\{\mathrm{S}_{\mathrm{i}} ; 1 \leq i \leq \mathrm{n}\right\}$. The forming temperature $T_{i, \text { gen }}$ of each parameter is reset by factor $s_{\max } / s_{i}$. The annealing time $k_{i}$ is also reset.

$$
\left\{\begin{array}{l}
T_{i, \text { gen }}\left(k_{i}\right)=\frac{s_{\max }}{s_{i}} T_{i, \text { gen }}\left(k_{i}\right) \\
k_{i}=\left[-\frac{1}{c} \log \left[\frac{T_{i, g e n}\left(k_{i}\right)}{T_{i, g e n}(0)}\right]\right]^{n}
\end{array}\right.
$$

At the same time, reset $T_{\text {accept }}(0)$ as final accepted cost function. Reset $\mathrm{T}_{\text {accept }}\left(k_{\alpha}\right)$ as $\mathrm{J}\left(\omega_{\text {best }}\right)$. The tempering time is reset by following equation.

$$
k_{\alpha}=\left[-\frac{1}{c} \log \left[\frac{T_{\text {accept }}\left(k_{\alpha}\right)}{T_{\text {accept }}(0)}\right]\right]^{n}
$$

(IV).The annealing process is proceeded at every $N_{\text {genera }}($ th $)$ generating point.

$$
\left\{\begin{array}{l}
k_{i}=k_{i}+1 \\
T_{i, \text { gen }}\left(k_{i}\right)=T_{i, \text { gen }}(0) \exp \left(-\mathrm{ck}_{i}^{\frac{1}{n}}\right)
\end{array}\right\} 1 \leq i \leq n
$$

and

$$
\left\{\begin{array}{l}
k_{\alpha}=k_{\alpha}+1 \\
T_{\text {accept }}\left(k_{\alpha}\right)=T_{\text {accept }}(0) \exp \left(-\mathrm{ck}_{\alpha}^{\frac{1}{n}}\right)
\end{array}\right\}
$$

The process ends if the parameters stay the same every continuously tempering and annealing time, or, the evaluation number of cost function reaches the minimum value, as well as the error be less than the given number. Otherwise returning to (II).

\section{RESULT AND DISCUSSION}

\subsection{Optimization Results}

The initial temperature is designated as $1{ }^{\circ} \mathrm{C}$. The convergence check interval is designated as 5 . The decreasing relative ratio of temperature parameter is designated as 1 . The temperature loss function drop ratio is designated as 1 . The allowed calculation failure time is 5. After 10112 times of iteration, the optimal value of the plate thickness is obtained in the factor design space while the peak sound pressure at driver's head is the minimum.

The plate thickness of the finite element model was chosen by the determined value of the plate thickness parameter. The body mass, structural modal and sound pressure distribution of the cab were calculated. The response values calculated by the approximation model and the finite element model are compared in table 3 .

Table 3. Comparison of response values

\begin{tabular}{|c|c|c|c|}
\hline & $\begin{array}{c}\text { Approximation } \\
\text { model }\end{array}$ & $\begin{array}{c}\text { Finite element } \\
\text { model }\end{array}$ & Error ratio \\
\hline$D / \mathrm{dB}$ & 76.58609 & 78.43 & $-2.35 \%$ \\
\hline$d r m s / \mathrm{dB}$ & 58.70524 & 59.02441 & $-0.54 \%$ \\
\hline$m / \mathrm{t}$ & 0.499967 & 0.498 & $0.39 \%$ \\
\hline$s f / \mathrm{Hz}$ & 24.03601 & 24.1824 & $-0.61 \%$ \\
\hline
\end{tabular}

According to Table 4, the error rate of the response calculated by the approximation model and finite element model is very small. It shows that the approximation model established by RBF neural network is of high accuracy, and the optimization result is of high reliability.

\subsection{Discussion}

The before and after the optimization value including the thickness parameter of the body plate, body mass, seventh rank modal frequency of the vehicle body, peak value of sound pressure and RMS value of the sound pressure at the driver's head were compared in this part. The results are shown in table 4 .

Table 4. Before and after optimization Comparison

\begin{tabular}{|c|c|c|}
\hline Variable & Before optimization & After \\
\hline$t_{1} / \mathrm{mm}$ & 0.8 & 2 \\
\hline$t_{2} / \mathrm{mm}$ & 0.7 & 0.77 \\
\hline$t_{3} / \mathrm{mm}$ & 0.9 & 0.94 \\
\hline$t_{4} / \mathrm{mm}$ & 0.8 & 0.5 \\
\hline
\end{tabular}




\begin{tabular}{|c|c|c|}
\hline$t_{5} / \mathrm{mm}$ & 0.9 & 1.58 \\
\hline$t_{6} / \mathrm{mm}$ & 0.9 & 0.58 \\
\hline$D / \mathrm{dB}$ & 82.58 & 78.43 \\
\hline$d r m s / \mathrm{dB}$ & 61.32021 & 59.02441 \\
\hline$m / \mathrm{t}$ & 0.4802 & 0.498 \\
\hline$s f / \mathrm{Hz}$ & 23.2706 & 24.1824 \\
\hline
\end{tabular}

According to the comparison, the peak value of the maximum sound pressure at the driver's head D is decreased by $4.15 \mathrm{~dB}$, and the RMS sound pressure is decreased by $2.3 \mathrm{~dB}$. The weight of the car body is only increased by $17.8 \mathrm{~kg}$, which can meet the requirements of lightweight design. The seventh rank modal frequency of the body is $24.1824 \mathrm{~Hz}$, which can avoid the radiation noise caused by the presence of a lower local mode in the car door caused by external excitation. In order to analyze the change of sound pressure in cab cavity at $20 \mathrm{~Hz} 200 \mathrm{~Hz}$, the before and after optimization A-level weighting acoustic pressure curve at the driver's head D were compared in figure 5.

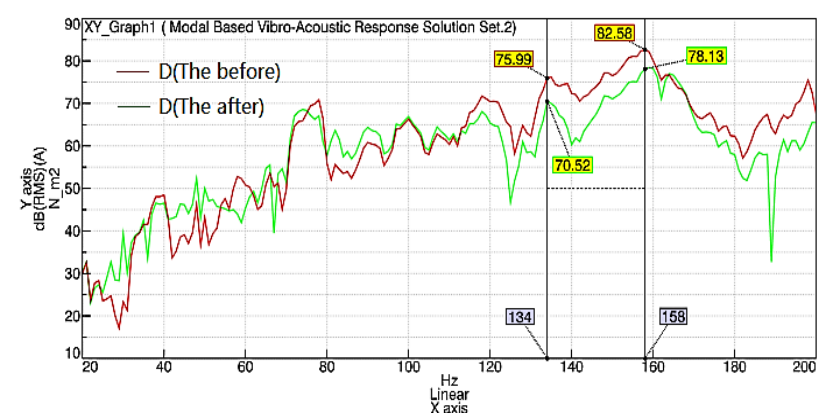

Fig. 5. A weighting pressure curves before and after optimization at driver's head

As shown in Figure 5, the sound pressure at the driver's head decreased significantly. The max sound pressure peak at $158 \mathrm{~Hz}$ is decreased by $4.45 \mathrm{~dB}$. The sound pressure peak at $134 \mathrm{~Hz}$ is decreased by $5.47 \mathrm{~dB}$. The measuring point sound pressure is decreased at different degree. It illustrates that the cab cavity noise is effectively decreased and the acoustic performance is improved through the optimization of body plate thickness.

\section{CONCLUSION}

(1) The optimal Latin hypercube sampling was used for acquiring sample data. The approximation model established by RBF neural network has the characteristics of small error and high precision.

(2) After optimization, the thickness parameter of the body plate, body mass, seventh rank modal frequency of the vehicle body, peak value of sound pressure and RMS value of the sound pressure were decreased at different degree. It illustrates that through the optimization of body plate thickness, the cab cavity noise is effectively decreased, and the acoustic performance is improved.

(3) Based on the established RBF neural network approximation model, the objective function was designated as the minimum of peak sound pressure at driver's head. Simulation results show that the adaptive annealing algorithm used for optimizing the plate thickness parameter to reduce the sound pressure of cab is a feasible and effective method.

\section{ACKNOWLEDGEMENTS}

This research is funded by Chongqing Research program of Basic Research and Frontier Technology (No. :cstc2015jycjBX0133) and National Natural Science Foundation of China(No. :51375519).

\section{REFERENCE}

1. Gonzalez A, Ferrer M, Diego M D, et al. Sound quality of low-frequency and car engine noises after active noise control. [J]. Journal of Sound \& Vibration, 2003, 265(3):663-679.

2. Liu Yu, Yu Fan, Liu Jiang. Modal analysis of vehicle ride acoustic-structure interaction. [J] Journal of noise and vibration control, 2005, 67(5):38-40.

3. Bai Shengyong, Qi Xiaoxiong, Ding Yulan. Acoustic modal analysis of passenger car. [J] Journal of Tongji University, 2000, 28(1):206-209.

4. Zang Xianguo. Structural acoustic performance optimization based on modal shape and response surface method. [D]Changsha: Hunan University, 2011.

5. Zhu Caichao, Qin Datong. Coupling analysis and control of car body structure vibration and interior noise. [J] Journal of Mechanical Engineering. 2002, 38 (8): 54-58.

6. Sung S, Chao S, Lingala H, et al. Structural-Acoustic Analysis of Vehicle Body Panel Participation to Interior Acoustic Boom Noise[C]// SAE 2011 World Congress \& Exhibition. 2011:650-657.

7. Qi Chang, Zhou Hong. Sound and vibration optimization of vehicle body panels based on the contribution of vehicle interior acoustic field. [J] Automotive Engineer, 2015, 37 (12):1438$1444,1432$.

8. SHEN Yanyou, JIA Mingpin, ZHU Lin. Research on noise analysis and noise reduction optimization of centrifugal leaf suction machine based on Latin hypercube sampling. [J] Journal of Vibration and shock. 2016, 35(15):93-97.

9. LI supping, HU qiguo, HU haibo, LUO tianhong. Vehicle's Low Frequency Noise Prediction and Body Panel's Acoustic Contribution Analysis. [J] Journal of noise and vibration control. 2016, 36(4):103-107.

10. Lai Yuyang. Parameter optimization theory and examples based on Isight. [M] BeiJing: Beijing University of Aeronautics and Astronautics Press.2012.

11. Ingber L. Adaptive simulated annealing (ASA): Lessons learned. [J]. Control \& Cybernetics, 2002, 25(1):33-54.

12. Liu P, Liu Y L, Fei-Hong Y U. Thin film optical parameters determination using adaptive simulated annealing algorithm [J]. Optical Instruments, 2008. 